\title{
Development and validation of an osteoporosis treatment questionnaire (OSTREQ) evaluating physicians' criteria in the choice of treatment
}

\author{
Polyzois Makras, ${ }^{1}$ Antonios Galanos, ${ }^{2}$ Stavroula Rizou, ${ }^{3}$ \\ Athanasios D. Anastasilakis, ${ }^{4}$ George P. Lyritis ${ }^{3}$
}

${ }^{1}$ Department of Endocrinology and Diabetes, 251 Hellenic Air Force \& VA General Hospital, Athens; ${ }^{2}$ Laboratory for the Research of the Musculoskeletal System, University of Athens, Athens; ${ }^{3}$ Hellenic Osteoporosis Foundation, Athens; ${ }^{4}$ Department of Endocrinology, 424 Military Hospital, Thessaloniki; Greece

\begin{abstract}
OBJECTIVE: This article describes the development and validation of the osteoporosis treatment questionnaire (OSTREQ), which is a physician-reported outcome tool aiming to evaluate physicians' criteria in the choice of osteoporosis treatment. DESIGN: The questionnaire -named OSTREQ- consisting of 17 questions that were divided into eight sections "Health Care System", "Patients' Preference in administration routes", "Usage, Cost", "Severity of Disease", "Treatment Efficacy", "Safety Profile", "Pharmaceutical Industry", affecting the decision and overall execution of a therapeutic approach, was developed by an expert panel and was later officially translated into English. In the second phase, orthopedic surgeons were asked to complete OSTREQ. Six indirect methods to evaluate validity were adopted: exploratory factor analysis, confirmatory factor analysis, subscale validity, known group validity, floor or ceiling effects, interpretability. To assess the reliability of the questionnaire, internal consistency validity as well as test-retest and parallel forms were calculated. RESULTS: One hundred seventy-two orthopedic surgeons were interviewed with an average period of experience in clinical practice of $\mathbf{1 0 . 5}$ years ( $\mathrm{SD} \pm \mathbf{8 . 9}$ years). The factors "Severity of Disease" and "Treatment Efficacy" were the most important in the choice of osteoporosis treatment, while the factor "Pharmaceutical Industry" had the least impact. The methodology of validation proved that the questionnaire possesses construct validity, discriminate ability, reliability, and sensitivity to change. CONCLUSIONS: OSTREQ represents a comprehensive and focused tool that, for the first time, assesses physicians' criteria in the choice of osteoporosis treatment. This tool could assist health care systems and pharmaceutical companies to be aware which parameters drive physicians' preferences regarding osteoporosis treatment.
\end{abstract}

Key words: Osteoporosis, Physicians, Questionnaire, Treatment, Validation

Address for correspondence:

Dr. Polyzois Makras, MD, Phd, Dept. of Endocrinology \& Diabetes, 251 Hellenic Air Force \& VA General Hospital, 3 Kanellopoulou street, 11525 Goudi, Athens, Greece; Tel.: +30 210 7463606, Fax: +30 210 9638501, E-mail address: makras@internet.gr

Received: 20-03-2016, Accepted: 01-06-2016 


\section{INTRODUCTION}

Osteoporosis is a generalized skeletal disorder and represents a major health issue worldwide with serious complications that may even be life-threatening, especially for the elderly. Furthermore, osteoporosis creates a significant economic burden. The International Osteoporosis Foundation (IOF) estimated the total costs resulting from osteoporotic fractures at $€ 37$ billion in 2010 for countries within the European Union. ${ }^{1}$ For Greece, the cost was $€ 680$ million and, based on anticipated demographic changes, is expected to increase to $€ 814$ million by 2025 . $^{2}$

The proper management of osteoporosis and its associated fractures is essential for maintaining high health care standards and quality of life for patients. Although the disease is traditionally defined as a Tscore of 2.5 standard deviations or more compared to young normal populations, the majority of fragility fractures occur in patients within the osteopenic and not the osteoporotic range. ${ }^{3,4}$ Therefore, the decision treatment should consider, in addition to the well-known bone mineral density (BMD) index, the individual fracture risk profile. Several clinical factors associated with increased fracture risk have been identified and assessment tools for the prediction of osteoporotic fractures have been developed. The most widely used is the FRAX model (https:// www.shef.ac.uk/FRAX). ${ }^{5}$

Different criteria for the initiation of anti-osteoporotic treatment have been set in each country. The FRAX model has been calibrated for the Greek population and has been incorporated in the national diagnostic and therapeutic guidelines. ${ }^{6}$ Bearing in mind that cost-effective intervention thresholds should ideally be country-specific, we recently established the thresholds considered cost-effective within the Greek setting. ${ }^{\text {? }}$

Individualized treatment is considered the ideal way of dealing with the disease. After establishing the need to initiate osteoporosis treatment, physicians are expected to choose the most suitable regimen for the patient based on medical history information, fracture risk assessment and previous anti-osteoporotic treatment. Moreover, the risk to benefit ratio should be constantly considered in the design and implementation of a chosen therapeutic approach. ${ }^{8}$ Apart from patients' characteristics, the choice to initiate, change or continue a specific therapeutic intervention could be influenced by a wide range of factors including physician-related issues, health care system rules and, probably, pharmaceutical industry support and promotion. Therefore, and given the multiple available choices for osteoporosis treatment, it is still unclear how physicians reach their decision on a specific regimen and which factors influence their choices.

In this study, we aimed to develop and validate a simple questionnaire in order to evaluate the factors that influence clinicians' decision to initiate, continue or change a regimen for osteoporosis treatment.

\section{METHODS}

In order to develop the questionnaire, 10 experts ( 4 orthopedic surgeons, 4 endocrinologists, and 2 rheumatologists), recognized for their significant contribution to the field of osteoporosis, were invited to assist in this project. Each expert was asked to list the 10 most important factors that drive his/her decision when choosing a specific regimen for osteoporosis treatment (including initiation, continuation or change of a regimen), and a list of 100 questions was subsequently developed. After excluding his/ her own 10 questions, each member of the panel was given the remaining 90 questions in order to evaluate each one as "necessary", "useful but not necessary" or "not necessary". Similar questions were merged and the content validity ratio for each question was estimated; all questions with a score between 0.7 and 1 were included in the final questionnaire. ${ }^{9}$ The final questionnaire consisted of 17 questions divided into eight sections detailing the issues affecting the decision on and overall execution of a therapeutic approach, namely "Health Care System", "Patients' Preferences regarding regimen's administration", "Usage", "Cost", "Severity of Disease", "Treatment Efficacy", "Safety Profile", and "Pharmaceutical Industry". Given that the study is aimed at developing and validating an "OSteoporosis TREatment Questionnaire", the acronym OSTREQ was created.

As the questionnaire was originally developed in Greek, a translation into English also took place in accordance with the existing guidelines. ${ }^{10}$ Specifically, two bilingual translators, one with a medical 
background, produced the English translation and reached a consensus on the result. Two other translators, totally blind to the original translation, translated back the English version of the questionnaire into Greek in order to ensure that the translated version reflected the same item content as the original. Next, the study committee reviewed all translations and reached a consensus on the pre-final version, which was tested on 30 orthopedic surgeons who completed the questionnaire and were also interviewed about the meaning of each question. Finally, the study committee, in coordination with the developers of the questionnaire, adapted the final version, which is presented herein (Figure 1).

In the second phase of the study, orthopedic surgeons were asked to complete OSTREQ. The only inclusion criterion was their exclusive occupation in private practice; their demographic and professional characteristics are shown in Table 1. Participants were recruited from all over Greece in a representative manner according to the distribution of the specialty of orthopedics within the Greek territory. The questionnaire was scored so that the higher the score in each question the more the doctor was positively influenced

\begin{tabular}{|c|c|c|c|c|c|}
\hline $\begin{array}{l}\text { Evaluation } \\
\text { of factors affecting treatment choices for osteop or osis }\end{array}$ & 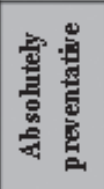 & 第竞 & 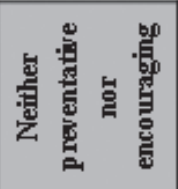 & 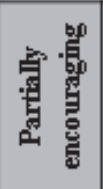 & 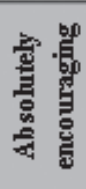 \\
\hline \multicolumn{6}{|l|}{ Health Care System } \\
\hline 1. Approval procedures and/or reimbursement policy of Health Care System & 1 & 2 & 3 & 4 & 5 \\
\hline \multicolumn{6}{|l|}{ Patients' pr ef er enc es r egar dingr egimen's a dministration } \\
\hline 2.Patient preferring oral administration & 1 & 2 & 3 & 4 & 5 \\
\hline 3. Patient preferring injectable administration & 1 & 2 & 3 & 4 & 5 \\
\hline 4.Patients' preferences regarding frequency of administration & 1 & 2 & 3 & 4 & 5 \\
\hline \multicolumn{6}{|l|}{ Usage } \\
\hline 5. Patients' capability in following required administration procedures & 1 & 2 & 3 & 4 & 5 \\
\hline 6. Procedures and requirements for the preservation of regimen & 1 & 2 & 3 & 4 & 5 \\
\hline \multicolumn{6}{|l|}{ Cost } \\
\hline 7. Cost for the Health Care System & 1 & 2 & 3 & 4 & 5 \\
\hline 8.Cost for the patient & 1 & 2 & 3 & 4 & 5 \\
\hline \multicolumn{6}{|l|}{ Severity of disea se } \\
\hline 9.Decreased bone mineral density & 1 & 2 & 3 & 4 & 5 \\
\hline 10. Risk of future osteoporotic fractures & 1 & 2 & 3 & 4 & 5 \\
\hline 11. Prevalent osteoporotic fractures & 1 & 2 & 3 & 4 & 5 \\
\hline \multicolumn{6}{|l|}{ Treatment efficacy } \\
\hline 12. Effect on reducing fracture risk & 1 & 2 & 3 & 4 & 5 \\
\hline 13. Effect on bone mineral density & 1 & 2 & 3 & 4 & 5 \\
\hline 14. Effect on patients' quality oflife & 1 & 2 & 3 & 4 & 5 \\
\hline \multicolumn{6}{|l|}{ Safety profile } \\
\hline 15. Possible adverse events & 1 & 2 & 3 & 4 & 5 \\
\hline \multicolumn{6}{|l|}{ Pharma ceutical industry } \\
\hline 16. Patients' program support & 1 & 2 & 3 & 4 & 5 \\
\hline 17. Physicians' program support & 1 & 2 & 3 & 4 & 5 \\
\hline
\end{tabular}

Figure 1. OSTREQ questionnaire. 
Table 1. Demographic and professional characteristics of participating physicians

\begin{tabular}{lc}
\hline Gender (male/female) n (\%) & $163(94.8 \%) / 9(5.2 \%)$ \\
Age (yrs) & $47.21 \pm 7.68$ \\
Yrs of specialty & $8(14)[1-43]$ \\
Patients per month & $200(207.5)[15-1000]$ \\
Osteoporotic patients per month & $40(56.5)[2-350]$ \\
\% osteoporotic patients per month & $22.2 \%(18)[3.3-75]$ \\
\hline
\end{tabular}

Footnote: With the exception of age (mean $\pm \mathrm{SD})$, all other parameters are presented as median (IQR) [min-max] due to the sample's distribution.

towards selection of a treatment. No specific treatment was evaluated as the participants were instructed to fill in the questionnaire bearing in mind all available anti-osteoporotic medications.

All questions were coded and scored and the completed questionnaires were included in the data set. Individual unanswered items were excluded from the analysis. All the data were entered, checked for missing values, and analyzed using the statistical programs SPSS version 17.0 (SPSS Inc., Chicago, IL, USA) and SAS version 7.0 (SAS Institute, Cary, $\mathrm{NC}, \mathrm{USA})$. Data are presented as means $( \pm \mathrm{SD})$. The normal distribution of the data was examined using the Kolmogorov-Smirnov test and P-P plots. Next, six indirect methods to evaluate validity were adopted: exploratory factor analysis; confirmatory factor analysis; subscale validity; known groups validity; floor or ceiling effects; interpretability. To assess the reliability of the questionnaire, the internal consistency validity as well as test-retest and parallel forms were calculated.

We chose not to describe the validity and reliability methods in the "Methods" section but to elaborate upon them in the "Results" section together with the outcome of analysis in order to facilitate and improve the readability of the paper and help the reader follow the methodology.

The study was approved by the local Institutional Review Board of 251 Hellenic Air Force \& VA General Hospital, Athens. All participants provided their written informed consent.

\section{RESULTS}

A total of 226 orthopedic surgeons were invited to participate: 172 responded positively and completed the questionnaire (response rate: $76.1 \%$ ). Their average period of experience in clinical practice was 10.5 years ( \pm 8.9 years).

The descriptive statistics of the completed questionnaires are presented in Table 2 and in supplementary Table 1. The responses to most of the questions were normally distributed. Skewness is a measure of asymmetry and a value of more than 1 or less than -1 indicates skewness in the data. Skewness values ranged between -2.09 and 0.6 ; only 2 questions had skewness lower than -1 , indicating skewed or non-

Table 2. Descriptive statistics initial assessment and reassessment

\begin{tabular}{|c|c|c|c|c|c|}
\hline & & Mean & SD & Min & Max \\
\hline \multirow{5}{*}{$\begin{array}{l}\text { Initial assessment } \\
(\mathrm{n}=172)\end{array}$} & Administration - Usage & 17.44 & 2.96 & 6.00 & 23.00 \\
\hline & Disease-Treatmenttt efficacy & 25.77 & 5.25 & 7.00 & 30.00 \\
\hline & Health care system-cost & 9.19 & 2.41 & 4.00 & 15.00 \\
\hline & Pharmaceutical industry & 7.19 & 1.67 & 3.00 & 10.00 \\
\hline & Total score & 62.32 & 7.83 & 35.00 & 79.00 \\
\hline \multirow{5}{*}{$\begin{array}{l}\text { Reassessment } \\
(\mathrm{n}=40)\end{array}$} & Administration-Usage & 18.43 & 2.04 & 14.00 & 22.00 \\
\hline & Disease-Treatmenttt efficacy & 27.18 & 1.96 & 24.00 & 30.00 \\
\hline & Health care system-cost & 8.23 & 1.76 & 6.00 & 13.00 \\
\hline & Pharmaceutical industry & 6.98 & 1.10 & 4.00 & 10.00 \\
\hline & Total score & 63.28 & 5.24 & 53.00 & 76.00 \\
\hline
\end{tabular}

SD: standard deviation. 
normal distribution of the responses to these questions.

In this particular group of physicians, the factors "Severity of Disease" and "Treatment Efficacy" were the most important in the choice of osteoporosis treatment, while the factor "Pharmaceutical Industry" had the least importance (Table 2). More specifically, in terms of each particular query, question 12 regarding the effect of an agent on reducing fracture risk had the higher score (4.51), followed by question 14 (4.42) regarding the effect on patients' quality of life, which included the parameters mobility, self-care, usual activities, pain/discomfort, and anxiety/depression (supplementary Table 1). By contrast, question 15 concerning the possible adverse events had the lowest impact on treatment choice (2.73), followed by question 7 (3.03) regarding the cost borne by the National Health System (supplementary Table 1).

Exploratory factor analysis (EFA) was conducted using the principal component extraction method with Varimax rotation to determine the factor structure of the 17 questions of the OSTREQ questionnaire. Questions with factor loadings $\geq 40$ (including values that rounded to 0.40 ) and those that did not load on more than one factor were retained. Questions not meeting these criteria were removed one at a time. Factor analyses were repeated until a solution was attained in which all questions included in the analysis met all criteria. ${ }^{11,12}$

The Bartlett Test of Sphericity was 1590 and was highly significant $(p<0.001)$. The Kaiser-Meyer-Olkin Measure of Sampling Adequacy was equal to 0.801, showing that the data were suitable for factor analysis. The 17 questions were analyzed via the maximum likelihood extraction method using a Varimax rotation. Four factors, with an eigenvalue of over 1 and questions factor loadings greater than or equal to 0 $\geq 40$, were identified. We used the scree test to determine the number of factors to retain and rotate, which again suggested a 4-factor solution. Only question 15 was excluded from the analysis because it was loaded on more than one factor and its loadings were not greater or equal to 0.40 . Finally, the EFA yielded 16 questions with a 4-factor solution, the eigenvalue for the first factor being 5.2, accounting for $30.4 \%$ of the variance, for the second factor it 2.9 , accounting for $17.1 \%$, for the third factor 2.1 , accounting for
$12.1 \%$ of the variance, and for the fourth factor 1.3, accounting for $7.8 \%$. Factor loadings, which are the correlation coefficients between the questions and the factor, ranged from 0.65 to 0.90 (Tables 3 and 4).

Confirmatory factor analysis (CFA) was used to examine and confirm the factor structure of the questionnaire as suggested by the EFA of the questionnaire. The CFA was carried out using the Analysis of Moment Structure (AMOS) Version 7.0. ${ }^{13}$ The sample size required for the CFA based on researchers' conventions ranged for the participants' ratio from 3:1 to as high as 12:1. Stable factor models can be found with samples as small as $100^{14}$ and with samples as small as 150 if 10 or more items load at 0.4 or higher. ${ }^{15}$ The OSTREQ consisted of 17 questions, thus our sample size of 172 is within the above guidelines. Rejecting or accepting a model was based on a number of global fit indices: chi-square tested the fit of the observed covariance matrix obtained under the constraints of the model; the root mean square error of approximation (RMSEA); the comparative fit index (CFI); the normed fit index (NFI); the goodness fit index (GFI); and the adjusted GFI (AGFI). Chi-square-degrees of freedom (d.f.) ratio $<2.0$, RMSEA $<0.06, \mathrm{CFI}>0.90$,

Table 3. Eigenvalues and explained variance

\begin{tabular}{ccc}
\hline Component & Eigenvalues & \% of Variance \\
\hline 1 & 5.17 & 30.41 \\
2 & 2.91 & 17.10 \\
3 & 2.06 & 12.14 \\
4 & 1.34 & 7.85 \\
5 & 0.84 & 5.51 \\
6 & 0.79 & 4.65 \\
7 & 0.64 & 3.75 \\
8 & 0.57 & 3.33 \\
9 & 0.50 & 2.94 \\
10 & 0.42 & 2.44 \\
11 & 0.37 & 2.19 \\
12 & 0.31 & 1.83 \\
13 & 0.30 & 1.76 \\
14 & 0.23 & 1.36 \\
15 & 0.19 & 1.13 \\
16 & 0.16 & 0.93 \\
17 & 0.12 & 0.68 \\
\hline
\end{tabular}


Table 4. Factor loadings

\begin{tabular}{lcccc}
\hline Items & Disease - Treatment Efficacy & Administration - Usage & Health Care System - Cost & Pharmaceutical Industry \\
\hline Q12 & 0.878 & & & \\
Q13 & 0.873 & & \\
Q11 & 0.868 & & \\
Q10 & 0.863 & & \\
Q14 & 0.846 & & \\
Q9 & 0.771 & 0.764 & \\
Q5 & & 0.737 & \\
Q6 & 0.730 & \\
Q4 & 0.712 & \\
Q3 & 0.654 & \\
Q2 & & 0.853 \\
Q8 & & 0.844 \\
Q7 & & 0.723 \\
Q1 & & \\
Q17 & & \\
Q16 & & & \\
\hline
\end{tabular}

Question 15 excluded from the analysis; all loadings below 0.4 are not presented.

Extraction Method: Principal Component Analysis. Rotation Method: Varimax with Kaiser Normalization

GFI $>0.85$, AGFI $>0.80$, NFI $>0.90$ indicate an acceptable fit. ${ }^{16-19}$

A 4-factor model was conducted by confirmatory factor analysis (Figure 2) yielding acceptable global fit indices. The resulting global fit indices $\mathrm{X}^{2}=113.23$, $\mathrm{p}<0.001$, chi-square-degrees of freedom (d. f.) ratio $=2.01, \mathrm{RMSEA}=0.062, \mathrm{CFI}=0.91, \mathrm{NFI}=0.85$, $\mathrm{GFI}=0.85, \mathrm{AGFI}=0.81$ showed that the 4 -factor solution proposed herein should be retained.

Convergent or criterion validity was not performed because the OSTREQ is the first questionnaire which examines the factors that clinicians take into consideration when choosing a particular regimen either to initiate or to continue or to change a treatment for osteoporosis.

Subscale validity was evaluated by examining the subscale correlations. There was a low correlation between the 4 factors of the OSTREQ questionnaire $(r<0.25)$, indicating uncorrelated factors as expected. Only the factor "Disease - Treatment Efficacy" had a low statistically significant correlation with the factors "Pharmaceutical Industry" $(r=0.201 \mathrm{p}=0.008)$ and "Administration - Usage" $(\mathrm{r}=0.285 \mathrm{p}<0.001)$ (supplementary Table 2).

Known groups validity of the OSTREQ questionnaire was examined in terms of the ability of the questionnaire to distinguish between subgroups of doctors on the basis of their time of clinical experience (less than 5 years vs $5-15$ years vs more than 15 years). The ANOVA model was used for the statistical analysis.

The OSTREQ questionnaire discriminated well between subgroups of physicians on the basis of their clinical experience. Only the "Administration - Usage" and "Disease - Treatment Efficacy" subscales tended to be lower in physicians with $15+$ years working experience compared with those with less than 5 years, and 5-10 years, respectively (Table 5).

Floor or ceiling effects are considered to be present if more than $15 \%$ of responders achieve the lowest or highest possible score, respectively. ${ }^{20}$ If floor or ceiling effects are present, it is likely that extreme items are missing at the lower or upper end of the scale, indicating limited content validity. As a consequence, physicians with the lowest or highest possible score 


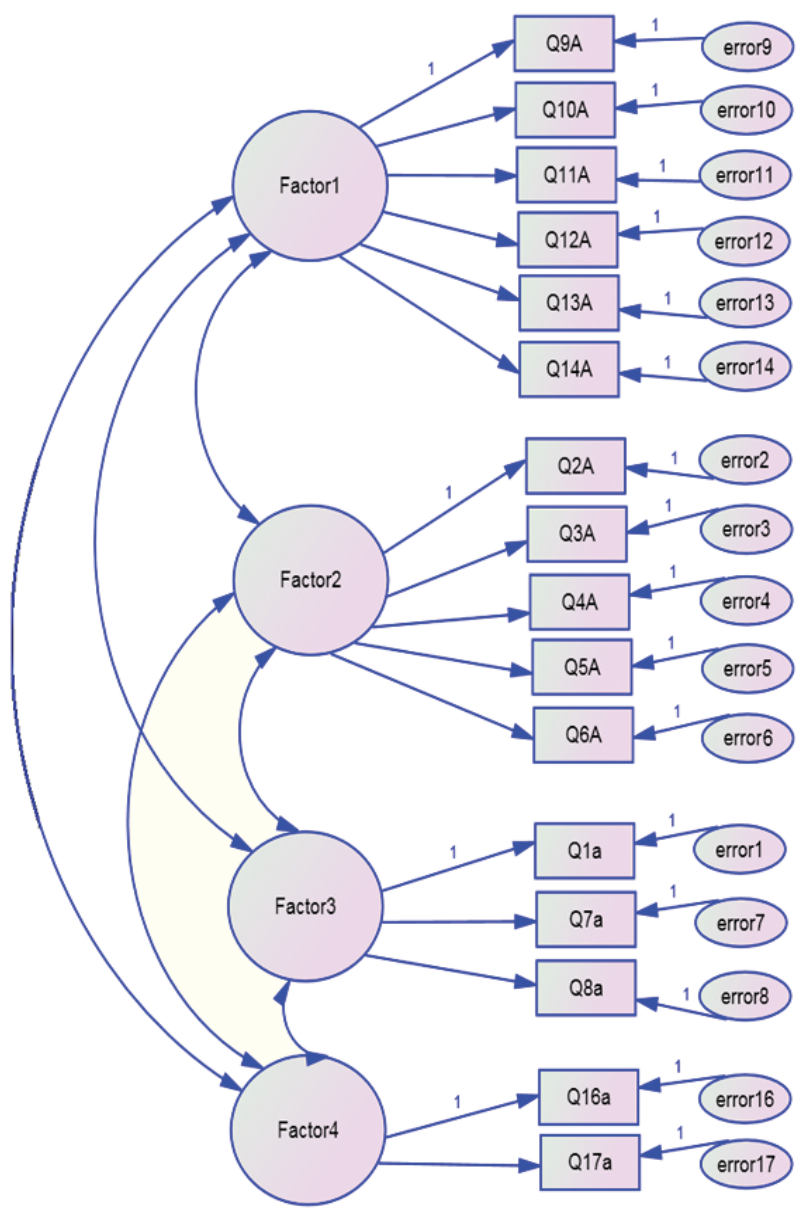

Figure 2. Confirmatory factor analysis, 4-factor structure.

cannot be distinguished from each other, thus reliability is reduced. Furthermore, the responsiveness is limited because changes cannot be measured in these physicians.

The percentage of physicians scoring at the lowest possible level of the scale and at the highest possible level for the "Administration - Usage", "Disease Treatment Efficacy", "Health Care System - Cost" and "Pharmaceutical Industry" factors were $0.6 \%$, $3.5 \%-0.6 \%, 26.2 \%-1.2 \%, 4.1 \%$ and $0.6 \%, 10.2 \%$, respectively. The critical value of $15 \%$ was surpassed only for the "Disease - Treatment Efficacy" factor which had a ceiling effect, thus it can conclusively be stated that there were no floor and ceiling effects for all factors.

Interpretability: The error associated with a single application of the OSTREQ was analyzed using the standard error of measurement (SEM). The SEM pro-
Table 5. Known-groups validity

\begin{tabular}{lllll}
\hline & Clinical status & N & Mean \pm SD & P value \\
\hline \multirow{2}{*}{ Administration - } & Below 5 years & 71 & $18.62 \pm 2.53$ & \\
Usage & 5-15 years & 46 & $19.04 \pm 2.52$ & $\mathbf{0 . 0 5 0}$ \\
& 15+ years & 54 & $17.17 \pm 2.92$ & \\
Disease - & Below 5 years & 71 & $27.98 \pm 2.49$ & \\
Treatment & 5-15 years & 46 & $27.23 \pm 4.56$ & $\mathbf{0 . 0 5 5}$ \\
Efficacy & 15+ years & 54 & $26.28 \pm 2.10$ & \\
& & & & \\
Health Care & below 5 years & 71 & $8.81 \pm 2.14$ & \\
System - Cost & 5-15 years & 46 & $8.65 \pm 1.96$ & 0.515 \\
& 15+ years & 54 & $8.11 \pm 2.42$ & \\
Pharmaceutical & Below 5 years & 71 & $7.12 \pm 1.29$ & \\
Industry & 5-15 years & 46 & $7.42 \pm 1.36$ & 0.468 \\
& 15+ years & 54 & $6.94 \pm 1.35$ & \\
& Below 5 years & 71 & $65.19 \pm 4.92$ & \\
Total & 5-15 years & 46 & $64.12 \pm 6.64$ & 0.346 \\
& 15+ years & 54 & $62.94 \pm 5.26$ & \\
\hline
\end{tabular}

vides an estimate of how reliably a scale estimates an individual's "true score", which is the score that would be obtained for the person if the scale were measured perfectly, without any error. Because clinicians are more interested in the error associated with an instant in time rather than over a short time interval, the SEM was calculated based on the alpha coefficient with the formula: SEM = standard deviation $\mathrm{x}$ [square root (1- Cronbach alpha)]. The SEM carries with it $68 \%$ CI. To achieve $90 \%$ CI, the SEM was multiplied by the $\mathrm{z}$ value associated with the $95 \% \mathrm{CI}(\mathrm{z}=1.96)$.

The error associated with the "Administration Usage", "Disease - Treatment Efficacy", "Health Care System - Cost" and "Pharmaceutical Industry" factors at a given point in time (SEM) were $0.21,1.15,0.48$, and 0.26 scale points, respectively (supplementary Table 3).

Internal consistency validity of the OSTREQ was determined by calculating the Cronbach alpha coefficient. ${ }^{21} \mathrm{~A}$ Cronbach alpha coefficient value of 0.7 indicates sufficient reliability for research purposes and suggests that items are interdependent and homogeneous in terms of the construct they measure. For clinical applications a $>0.8$ is desirable. ${ }^{22}$ The 
internal consistency of the 17 items of the OSTREQ questionnaire was measured with Cronbach's alpha and yielded a value from 0.780 for the factor "Disease - Treatment Efficacy" to 0.93 for the factor "Administration - Usage", which indicate excellent internal consistency; the items are interdependent and homogeneous in terms of the construct they measure (supplementary Table 4).

Test-retest reliability (stability) indicates the stability of patients' response in time and it was determined by calculating ICC (intraclass correlation coefficient: the error in measurements as a proportion of the total variance) between the total scores of the initial assessment of the OSTREQ and the total scores of the reassessment after 3 days. Because this coefficient does not correct for systematic differences and agreement by chance, the scores of the 2 assessments were tested for systematic differences by using the paired $t$-test. $23-25$

The paired samples t-test between initial assessment and reassessment of all factors indicated no statistically significant differences. ICC between initial assessment and reassessment of the test ranged between 0.890 and $0.925(\mathrm{p}<0.001)$. The above results of stability indicated that OSTREQ's factors were remarkably consistent between the two occasions (supplementary Table 5).

Parallel forms reliability is a measure of reliability obtained by administering different versions of the OSTREQ questionnaire (both versions must contain questions that probe the same construct, skill, knowledge base, etc.) to the same group of individuals. The scores from the two versions can then be correlated in order to evaluate the consistency of results across alternate versions. ${ }^{23-25}$ The reliability of the parallel forms was examined using a sample of 40 random physicians. An example of the form of questions is presented in supplementary Figure 1. The scores from the two different versions of the OSTREQ questionnaire were very highly correlated for all factors $(\mathrm{r}$ $>0.9890$ ), which proved the consistency of results across alternate versions.

\section{DISCUSSION}

Several questionnaires have been developed to assess the risk of fracture and the necessity of treatment in patients with decreased bone mass. ${ }^{26-28}$ Furthermore, there are studies evaluating questionnaires based on patients' preferences regarding osteoporosis treatment. ${ }^{29-32}$ However, all these tools are exclusively addressed to patients. In the present study, we aimed to develop what is, to the best of our knowledge, the first questionnaire evaluating the factors that influence the physician's decision to initiate, continue or change a regimen for osteoporosis treatment.

Our questionnaire was completed by physicians of the same specialty. In contrast to other countries, orthopedic surgeons represent the specialty with the highest percentage of prescriptions for osteoporosis treatment in Greece. The participants were representatively selected from all regions of Greece in order to avoid local or regional biases in their responses. From their ranking, it is evident that questions associated with the severity of the disease and the efficacy of each treatment weigh the most in a physician's decision to administer a specific treatment, while cost, system approval procedures and pharmaceutical industry support are considered of less importance. In addition, it seems that the possibility of adverse events is not a major issue of concern among this group of physicians, indicating that osteoporosis treatment is still regarded as a reasonably safe therapeutic intervention with minimum and rare unfavorable events. More experienced physicians tended to value patients' preferences and difficulties in the procedures of treatment less than physicians with fewer years of experience. In addition OSTREQ failed to discriminate between subgroups of physicians on the basis of their clinical experience as concerns the subscales of "Health Care System - Cost" and "Pharmaceutical Industry". However, this phenomenon is clinically relevant as clinical experience is obviously important in terms of administration and usage of medication as well as regarding factors such as the disease per se and the treatment used. Factors such as health care system, cost, and pharmaceutical industry are expected to have a universal impact on physicians and this was proved with the analysis of "known group's validity" in this group of doctors.

The same questionnaire could be completed by other specialties involved in the management of osteoporosis, e.g. endocrinologists, rheumatologists, etc.; 
in that case, the recorded responses could be considerably different from those recorded by the orthopedic surgeons in the present study. Furthermore, physicians from different countries could respond quite differently based on their culture and professional habits as well as their national guidelines. However, this fact does not negate the validation of the instrument, which was adequately designed and performed very satisfactorily. To our knowledge, there are no data regarding the physicians' attitudes in other countries, as information is only given after the implementation of national guidelines and health care system rules and not as a consequence of personal preferences and decisions; OSTREQ might be helpful to clarify the parameters affecting the physicians' decision in every country and specialty and thereby guide both medical education and health care policies.

The present tool was developed as a general osteoporosis treatment questionnaire; however, it could be used easily and with minimal modifications to evaluate physicians' opinion on a specific antiosteoporotic agent. Finally, our questionnaire could serve as the basis for the development of physician-addressed questionnaires evaluating other diseases and conditions, e.g. diabetes mellitus, arterial hypertension, etc.

In conclusion, we developed and validated a general osteoporosis treatment questionnaire that could provide assessment of the criteria that physicians take into consideration when they decide to implement a regimen for osteoporosis. This tool could assist health care systems and pharmaceutical companies understand which parameters drive physicians' choices regarding the treatment of osteoporosis.

\section{REFERENCES}

1. Hernlund E, Svedbom A, Ivergard M, et al, 2013 Osteoporosis in the European Union: medical management, epidemiology and economic burden. A report prepared in collaboration with the International Osteoporosis Foundation (IOF) and the European Federation of Pharmaceutical Industry Associations (EFPIA). Arch Osteoporos 8: 136.

2. Svedbom A, Hernlund E, Ivergard M, et al, 2013 Epidemiology and economic burden of osteoporosis in Greece. Arch Osteoporos 8: 83-90.

3. Siris ES, Miller PD, Barrett-Connor E, et al, 2001 Identification and fracture outcomes of undiagnosed low bone mineral density in postmenopausal women: results from the National Osteoporosis Risk Assessment. JAMA 286: 2815-2822.

4. Kanis JA, Johnell O, Oden A, et al, 2000 Risk of hip fracture according to the World Health Organization criteria for osteopenia and osteoporosis. Bone 27: 585590.

5. WHO Fracture Risk Assessment Tool. http://www.shef. ac.uk/FRAX/?lang=en. Accessed 7 November 2015.

6. Makras P, Vaiopoulos G, Lyritis GP, Greek National Medicine A, 20122011 guidelines for the diagnosis and treatment of osteoporosis in Greece. J Musculoskelet Neuronal Interact 12: 38-42.

7. Makras P, Athanasakis K, Boubouchairopoulou N, et al, 2015 Cost-effective osteoporosis treatment thresholds in Greece. Osteoporos Int 26: 1949-1957.

8. Lecart MP, Reginster JY, 2011 Current options for the management of postmenopausal osteoporosis. Expert Opin Pharmacother 12: 2533-2552.

9. Lawshe C, 1975 A quantitative approach to content validity. Personnel Psychology 28: 563-575.

10. Beaton DE, Bombardier C, Guillemin F, Ferraz MB, 2000 Guidelines for the process of cross-cultural adaptation of self-report measures. Spine (Phila $\mathrm{Pa} 1976$ ) 25: 3186-3191.

11. Gorgush RL 1978 Psychometric Theory. USA: McGraw - Hill.

12. Harman HH 1967 Modern Factor Analysis. Chicago: University of Chicago Press.

13. Arbuckle JL 2006 AMOS 7.0 user's guide. Chicago, IL.

14. Fabrigar LR, Wegener DT, Mac Callum RC, Strahan EJ, 1999 Evaluating the use of exploratory factor analysis in psychological research. Psychological Methods 4: 272-299.

15. Guadagnoli E, Velicer WF, 1988 Relation to sample size to the stability of component patterns. Psychological Bulletin 103: 265-275.

16. Hu L, Bentler PM, 1999 Cutoff criteria for fit indexes in covariance structure analysis: conventional criteria versus new alternatives. Structural Equation Modelling 6: 1-55.

17. Byrne BM 1989 A primer of LISREL: Basic applications and programming for confirmatory analytic models. New York: Springer-Verlag.

18. Mc Donald RP, Marsh HW, 1990 Choosing a multivariate model: noncentrality and goodness of fit Psychological Bulletin 107: 247-255.

19. Bollen KA 1989 Structural equations with latent variables. New York: John Wiley \& Sons.

20. McHorney CA, Tarlov AR, 1995 Individual-patient monitoring in clinical practice: are available health status surveys adequate? Qual Life Res 4: 293-307.

21. Cronbach LJ, 1951 Coefficient alpha and the internal structure of test. Psychometrica 16: 297-334.

22. Higgins PA, Straub AJ, 2006 Understanding the error of our ways: mapping the concepts of validity and 
reliability. Nurs Outlook 54: 23-29.

23. Bland JM, Altman DG, 2002 Statistics Notes: Validating scales and indexes. BMJ 324: 606-607.

24. Boynton PM, 2004 Administering, analysing, and reporting your questionnaire. BMJ 328: 1372-1375.

25. Brink TL 2004 Questionnaires. Practical hints on how to avoid mistakes in design and interpretation. Chesterfield: Heuristic Books.

26. Koh LK, Sedrine WB, Torralba TP, et al, 2001 A simple tool to identify asian women at increased risk of osteoporosis. Osteoporos Int 12: 699-705.

27. McDonough CM, Grove MR, Elledge AD, Tosteson AN, 2012 Predicting EQ-5D-US and SF-6D societal health state values from the Osteoporosis Assessment Questionnaire. Osteoporos Int 23: 723-732.

28. Naegeli AN, Nixon A, Burge R, et al, 2014 Development of the Osteoporosis Assessment Questionnaire--physical Function (OPAQ-PF): an osteoporosis-targeted, patientreported outcomes (PRO) measure of physical function. Osteoporos Int 25: 579-588.
29. Gold DT, Horne R, Coon CD, et al, 2011 Development, reliability, and validity of a new Preference and Satisfaction Questionnaire. Value Health 14: 1109-1116.

30. Freemantle N, Satram-Hoang S, Tang ET, et al, 2012 Final results of the DAPS (Denosumab Adherence Preference Satisfaction) study: a 24-month, randomized, crossover comparison with alendronate in postmenopausal women. Osteoporos Int 23: 317-326.

31. Hadji P, Ziller V, Gamerdinger D, et al, 2012 Quality of life and health status with zoledronic acid and generic alendronate--a secondary analysis of the Rapid Onset and Sustained Efficacy (ROSE) study in postmenopausal women with low bone mass. Osteoporos Int 23: 2043-2051.

32. Lee S, Glendenning P, Inderjeeth CA, 2011 Efficacy, side effects and route of administration are more important than frequency of dosing of anti-osteoporosis treatments in determining patient adherence: a critical review of published articles from 1970 to 2009 . Osteoporos Int 22: 741-753. 